Saudi Journal of Oral and Dental Research

Abbreviated Key Title: Saudi J Oral Dent Res

ISSN 2518-1300 (Print) |ISSN 2518-1297 (Online)

Scholars Middle East Publishers, Dubai, United Arab Emirates

Journal homepage: https://saudijournals.com

\title{
i-PRF a Boon In Regenerative Periodontal Therapy: A Case Report of 1- Year Follow-Up
}

Dr. Sharma Manohar H, Dr. Gujjari Sheela Kumar, Dr. Medha Sharma*, Dr. Nair Uma P

BDS, JSS Dental College \& Hospital Mysuru, India

DOI: $10.36348 /$ sjodr.2020.v05i12.003

| Received: 26.11.2020 | Accepted: 09.12.2020 | Published: 16.12 .2020

*Corresponding author: Dr. Medha Sharma

\section{Abstract}

Wound healing involves a complex cascade of events, ordered and complex, involving many types of cells, which are driven by the release of soluble mediators and signals able to influence these circulating cells and cause them to home back to damaged tissues. Platelets proved themselves to be an important cells regulating haemostasis phases through the vascular occlusion and facilitating fibrin clot formation. This fibrin scaffold, devoid of any cytotoxic property, is obtained from $9 \mathrm{ml}$ of patient's blood, after centrifugation performed with a centrifuge and the use of gel-free vials; it contains a variety of blood cells- including platelet, B and T lymphocytes, monocytes, stem cells and neutrophilic granulocytes- other than growth factors. L- PRF (leukocyte-PRF) and its derivatives (A-PRF, i- PRF and so on). There is an abundance of literature on the use of injectable PRF (i-PRF) on dental implants with successful results. Therefore, in this case report i-PRF was used as regenerative alloplastic material for an infrabony defect. At 9 months follow-up, there was a reduction in pocket depth and radiographic evidence of bone fill was also noted.

Keywords: Injectable PRF, grade II furcation, sticky bone, infrabony defect, bone graft, regeneration, periodontal guided tissue regeneration.

Copyright () 2020 The Author(s): This is an open-access article distributed under the terms of the Creative Commons Attribution 4.0 International License (CC BY-NC 4.0) which permits unrestricted use, distribution, and reproduction in any medium for non-commercial use provided the original author and source are credited.

\section{INTRODUCTION}

Regenerative periodontal therapy aims at restoring the tooth supporting structures that has been lost due to periodontal disease or injury. Complete regeneration of periodontal tissues following periodontal disease has always been a challenge for the clinician. From last three decades there have been many advancements in the innovative methods and the materials for regenerative periodontal therapy, such as Guided Tissue Regeneration with or without bone graft, active biomaterials [1] and Platelet concentrates (PC) [platelet-rich plasma (PRP) and platelet-rich fibrin $(\mathrm{PRF})]$.

PC have come a long way since its first appearance in 1954 to the T-PRF, A-PRF and i-PRF introduced recently [2]. The first generation platelet concentrates are platelet-rich plasma (PRP). The clinical efficacy of the PRP was discovered in early 1990s when new "biological glues" were being discovered. These are produced using a specific centrifugation protocol together with anti-coagulants [3]. PRP was first described for clinical use in 1999 to enhance osseointegration for tooth implants well after specific individual growth factors had already been identified for healing of various wound and injury types [4]. The PRP is usually prepared by centrifugation of whole blood collected in a sterile container using citrate-based anticoagulant. The centrifugation can be a one-step or two-step protocol. After final centrifugation, a platelet pellet is formed which is resuspended in a small volume of platelet-poor plasma. Further, the platelets in PRP are usually activated by addition of various activators (calcium chloride, thrombin, chitosan, and batroxobin) for release of growth factors [5].

Then was introduced the second generation platelet concentrates, Platelet-rich fibrin (PRF), as proposed by Choukroun et al., is a leucocyte- and platelet rich fibrin matrix, a further development of PRP but without addition of anticoagulants. PRF was first developed for use in the field of oral and maxillofacial surgery, in France in the year 2001. A sample of blood is collected from patient without anticoagulant in $10 \mathrm{ml}$ tubes which are immediately centrifuged at a rate of $3000 \mathrm{rpm}$ for $10 \mathrm{~min}$ [6].

Recently, a lower centrifugation protocol of PRF production, so called injectable-PRF (i-PRF) was developed [7]. i-PRF remains liquid for approximately 
15 min. After application, it immediately coagulates into a PRF clot rich with blood-derived growth factors, which constantly release over 10-14 days. i-PRF has advantage in higher number of leukocyte as far as it favours more growth factor release and thus tissue wound healing. Mourão et al., 2015 described a technique to obtain an injectable form of PRF called iPRF. In this technique a short centrifugation for $2 \mathrm{~min}$ at $3300 \mathrm{rpm}$ gave an orange colour fluid which can be injected or mixed with bone graft to give a well agglutinated "steak" for bone grafting [8]. A new concept of fabricating growth factors-enriched bone graft matrix (also known as "sticky bone") using autologous fibrin glue has been demonstrated since last decade. Sticky bone helps in stabilization of bone graft which helps in acceleration of tissue healing and it also minimizes bone loss during healing phase [9]. Few studies have demonstrated that i-PRF has excellent biocompatibility, induce a significant number of cell migration and proliferation and when compared to PRP it promoted more osteogenic differentiation [10].

Keeping these advantages in mind, the aim of this case report was to describe the efficiency of i-PRF used with bone graft (sticky bone) in the treatment of wide intra-bony defect and Grade II furcation defect.

\section{Case Report}

A systemically healthy 31 year old male patient reported to the Department of Periodontology, JSS Dental College and Hospital, Mysuru, Karnataka with the chief complaint of pain in his lower left back teeth region. No extra oral findings were seen. On intraoral examination in the distal aspect of 36 , a periodontal pocket measuring $11 \mathrm{~mm}$ with Grade I mobility and bleeding on probing was noted (Figure-1). There was no periodontal pockets, mobility or loss of attachment in the rest of the dentition. Radiographic evaluation of 36 revealed vertical bone defect extending until the middle one-third of root (Figure-2). The tooth vitality was checked and the tooth was found to be vital. The possibility of trauma from occlusion was ruled out. A primary diagnosis of Chronic Generalized Gingivitis with Localized Periodontitis was made on the basis of clinical evaluation and radiographic findings. The treatment plan was devised with the main aim to preserve the tooth by treating the periodontal condition. The treatment started with Phase I Periodontal treatment which included Oral hygiene instructions, Scaling and Root Planing. The patient was recalled after 4 weeks for check-up. On examination it was noted that the periodontal pocket and attachment loss was still persistent. Therefore the decision was taken to perform regenerative periodontal therapy to reconstruct the lost periodontal structure. Patient was informed about the procedure and an informed surgical consent was taken from the patient prior to the surgery.

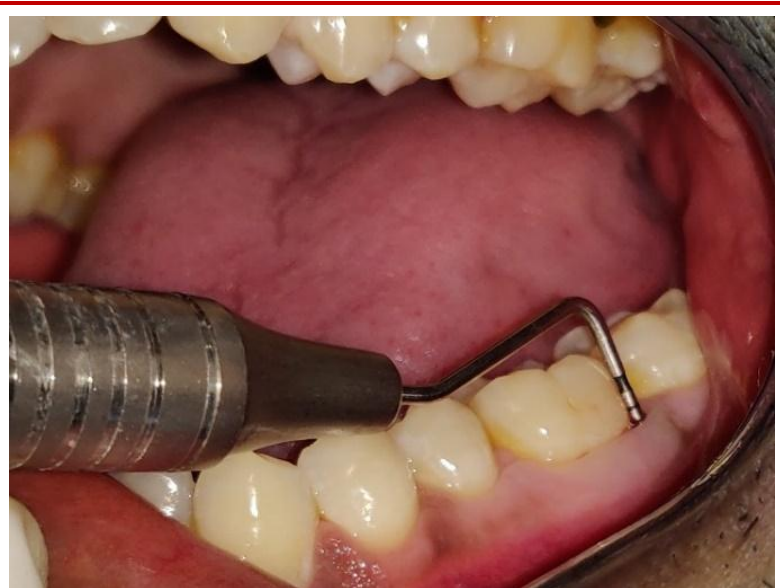

Fig-1: Pre-op probing depth

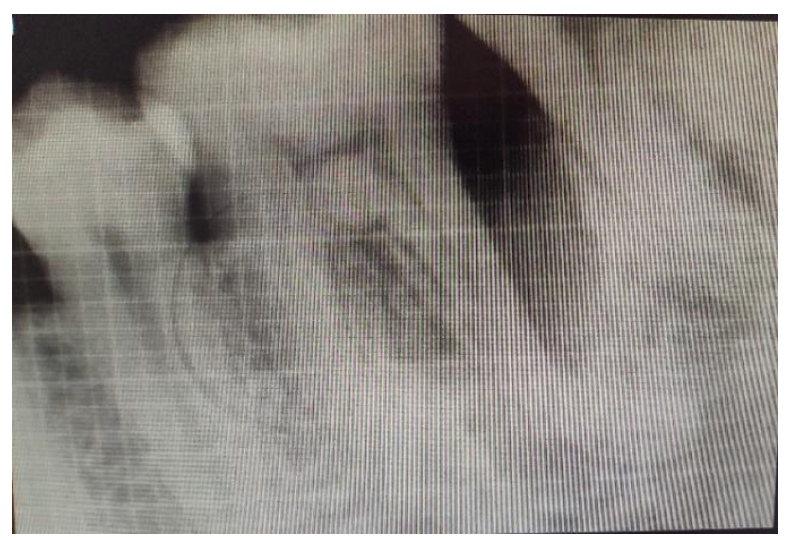

Fig-2: Pre-op radiographic analysis

$2 \%$ lignocaine with 1:80,000 adrenaline local anaesthesia was administered to the site, following which a full thickness mucoperiosteal flap was reflected and open flap debridement was done (Figure-3). After debridement a one wall bone defect of $11 \mathrm{~mm}$ depth and a grade II furcation of $3 \mathrm{~mm}$ depth was noted (Figure-4). The bone defect was filled with Sticky bone [Bio-oss* (bone graft material) and i-PRF (Figure-5). i-PRF was prepared by collecting the blood sample in two $10 \mathrm{ml}$ test tubes without anticoagulant which was then centrifuged at $700 \mathrm{rpm}$ for $3 \mathrm{~min}$ (60G) at room temperature in a Dentifuge. The upper liquid layer was collected as i-PRF. PerioCol-GTR membrane (sterile collagen periodontal membrane) was placed as a barrier membrane around the defect over the Sticky bone (Figure-6). The flap was approximated and sutured (Figure-7). The sutures were removed after a week (Figure-8) and patient was kept on maintenance therapy, which included a recall visit every 2 weeks for first 1-month, followed by every 3 weeks for 9 months postoperatively. 


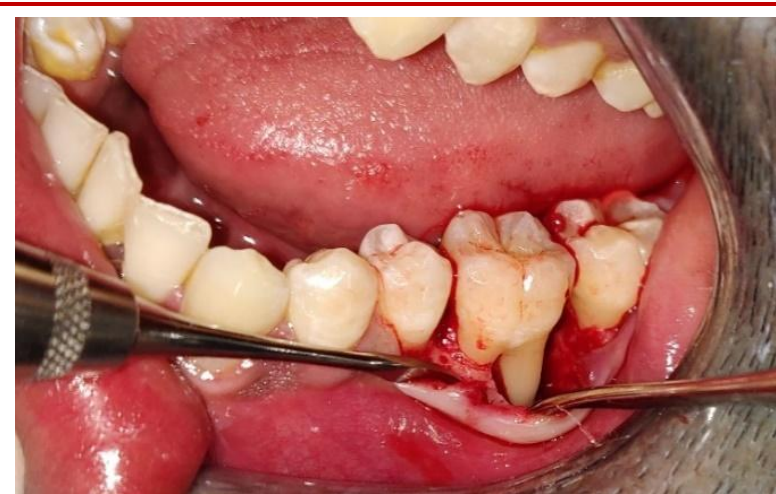

Fig-3: Flap Reflection

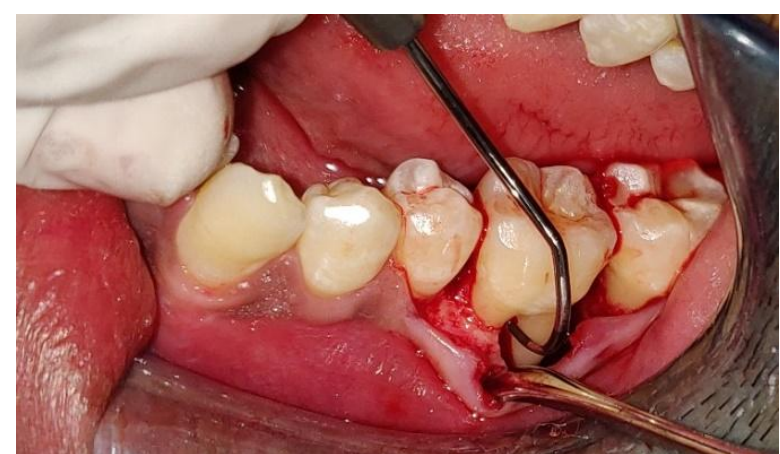

Fig-4: Measurement of furcation defect using Naber's Probe

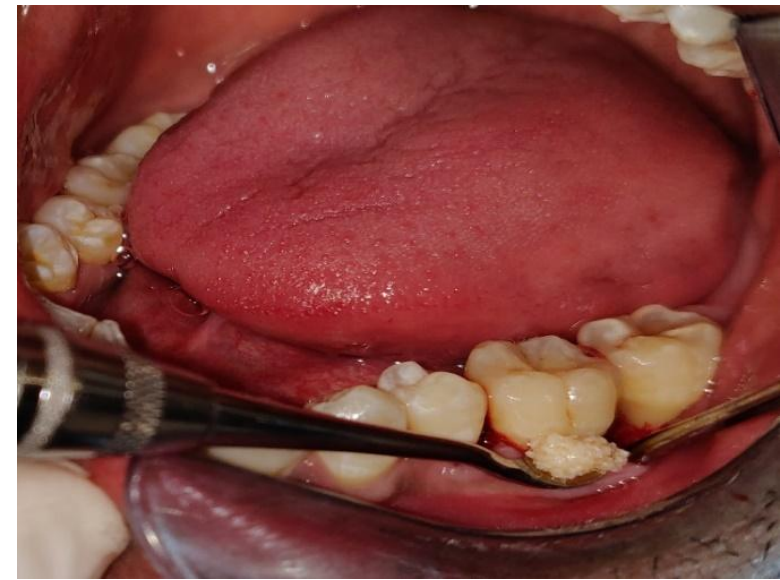

Fig-5: Sticky bone placement

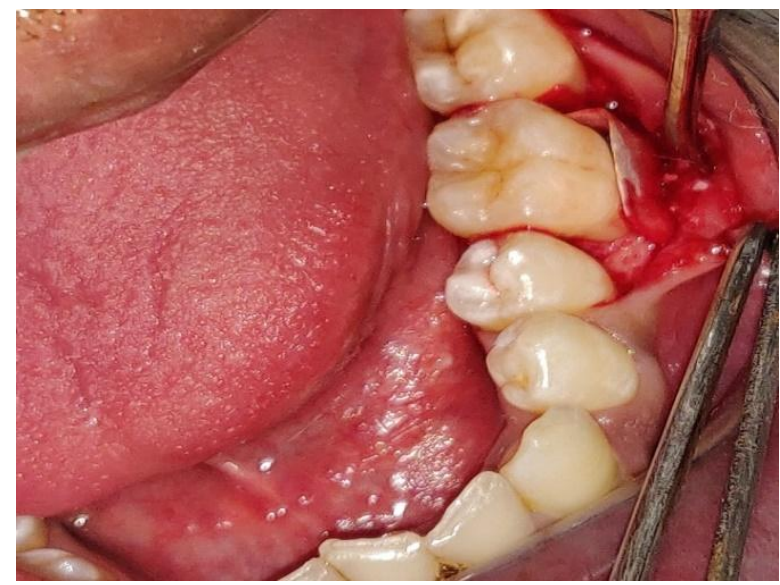

Fig-6: Collagen GTR membrane placement

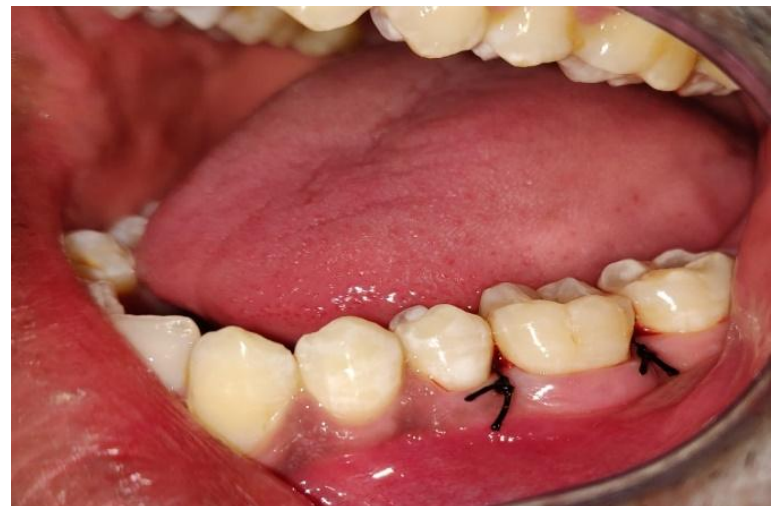

Fig-7: Suturing done

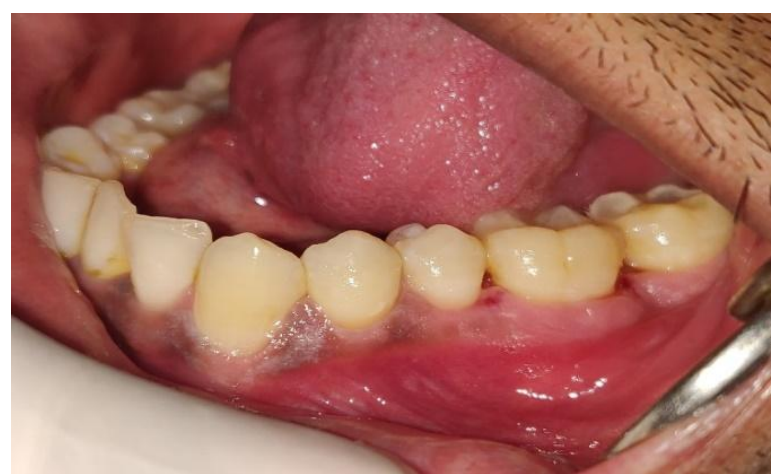

Fig-8: 2 weeks post-op

At the end of 3 months, there was a clinical attachment gain by $4 \mathrm{~mm}$, reduction in pocket depth by $6 \mathrm{~mm}$ (Figure-9), and at the end of 9 months bone fill was noted in the intraoral periapical radiographs (Figure-10). There was no mobility noted at each follow-up visits.

The healing could have happened through reattachment or regeneration. To confirm this histological analysis required which was not possible due to ethical concerns. Since bone fill was noted, the healing could be by repair or regeneration. Finally the patient was comfortable and satisfied with the treatment outcomes. Further, patient is kept under maintenance phase. Satisfactory healing and bone fill was noted after 1 year follow-up (Figure-11).

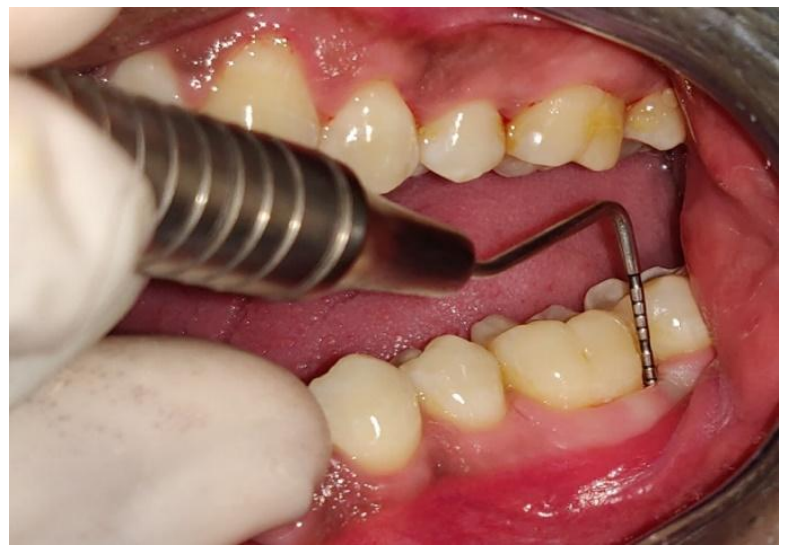

Fig-9: 9 months follow up; probing Depth 


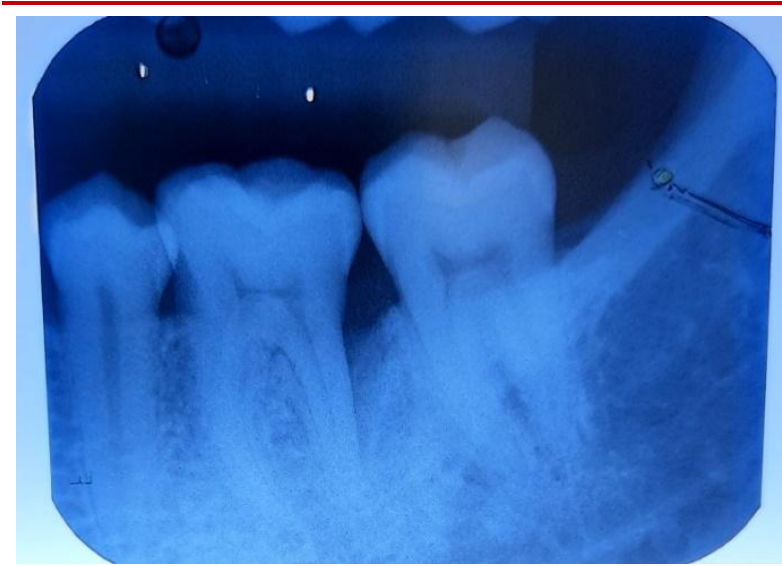

Fig-10: 9 months follow-up radiographic analysis

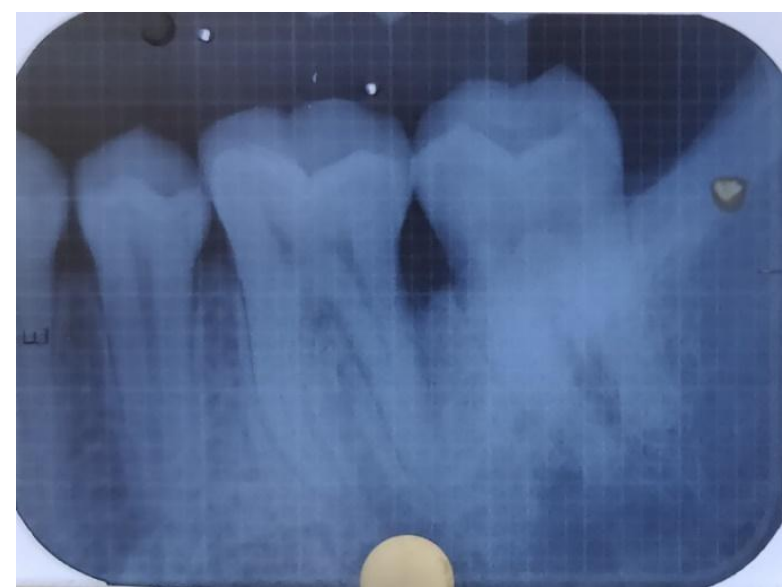

Fig-11: 12 months follow-up radiographic analysis

\section{DiscuSSION}

The goal of periodontal regenerative therapy is to completely restore the lost supporting periodontal apparatus of the tooth which has been lost due to injury. It is characterized by formation of new alveolar bone, new periodontal ligament, new cementum with inserting collagen fibers. Many treatment has been suggested for this therapy such as conventional, surgical and non-surgical [11].

There have been various advances in the field periodontal regeneration in the past few decades which helps to improve clinical outcomes in infrabony defects and class II furcation defects such as: use of various surgical techniques with bone graft, root surface demineralization, Guided tissue regeneration (GTR), growth and differentiation factors(PRP and PRF), combinations of these $[12,13]$.

The use of platelet concentrates including PRP and PRF which both utilize supra-physiological doses of autologous growth factors derived from the patient's own blood further capable of speeding tissue regeneration [14]. Concerns were made in PRP due to presence of anti-coagulants, therefore many advancements have been made possible due to recent findings by Ghanaati et al., who introduced "low speed concept" for blood centrifugation since lower centrifugation speeds were shown to contain higher numbers of cells including leukocytes prior to the formation of a fibrin clot. ${ }^{15}$ Leucocytes are the immune cells having vast property in tissue regeneration. It recruits and directs various cells during wound healing process [16].

Bone graft in combination with a barrier membrane have shown to increase the regenerative outcomes. Filling the defect with sticky bone and covering it with a barrier membrane have also shown to accelerate the bone formation and wound healing. iPRF with bone graft (sticky bone) has its own body and can be easily moulded into the required shape, thus aiding in its easy handling and also in preventing dispersion. ${ }^{17}$ Studies have shown that even following 10 days, an additional release of growth factors could still be expected from i-PRF whereas PRP had basically dissolved entirely after 10 days [18]. Hence in this study i-PRF with bone graft and sterile collagen periodontal membrane was used for regeneration.

As described by Sculean et al., [13] in the systematic review that most studies have demonstrated superior histologic healing following the combination of barrier membranes and grafting materials than following open flap debridement, hence in this case with the use of sticky bone, GTR membrane was also placed for better outcome.

The results of this report shows that with the use of i-PRF and bone graft material (sticky bone) along with a barrier membrane, it was possible to regenerate wide infrabony defects.

\section{CONCLUSION}

i-PRF showed the potential for regeneration since it has number of growth factors which are responsible for tissue regeneration capable of inducing fibroblast behaviour. Future studies are required to know the potential of i-PRF as a regenerative material.

\section{Acknowledgements: Nil}

Conflicts of Interest: The authors of the study have reported no conflict of interest.

\section{REFERENCES}

1. Garrett, S. (1996). Periodontal regeneration around natural teeth. Annals of periodontology, 1(1), 621-666.

2. Agrawal, A. A. (2017). Evolution, current status and advances in application of platelet concentrate in periodontics and implantology. World journal of clinical cases, 5(5), 159.

3. Kyyak, S., Blatt, S., Pabst, A., Thiem, D., AlNawas, B., \& Kämmerer, P. W. (2020). Combination of an allogenic and a xenogenic bone 
substitute material with injectable platelet-rich fibrin-A comparative in vitro study. Journal of Biomaterials Applications, 0885328220914407.

4. Marko B, Ryan D, Yvette U. Essentials of Regenerative Medicine In Interventional Pain Management. Chapter 8, Prp: History, Mechanism Of Action, Preparation And Clinical Applications.

5. Jain, A., Bedi, R. K., \& Mittal, K. (2015). Plateletrich plasma therapy: a novel application in regenerative medicine. Asian journal of transfusion science, 9(2), 113-114.

6. Saluja, H., Dehane, V., \& Mahindra, U. (2011). Platelet-Rich fibrin: A second generation platelet concentrate and a new friend of oral and maxillofacial surgeons. Annals of maxillofacial surgery, 1(1), 53-57.

7. Ghanaati, S., Booms, P., Orlowska, A., Kubesch, A., Lorenz, J., Rutkowski, J., ... \& Choukroun, J. (2014). Advanced platelet-rich fibrin: a new concept for cell-based tissue engineering by means of inflammatory cells. Journal of Oral Implantology, 40(6), 679-689.

8. Mourão, C. F. D. A. B., Valiense, H., Melo, E. R., Mourão, N. B. M. F., \& Maia, M. D. C. (2015). Obtention of injectable platelets rich-fibrin (i$\mathrm{PRF}$ ) and its polymerization with bone graft. Revista do Colégio Brasileiro de Cirurgiões, 42(6), 421-423.

9. Wang, X., Zhang, Y., Choukroun, J., Ghanaati, S., \& Miron, R. J. (2018). Effects of an injectable platelet-rich fibrin on osteoblast behavior and bone tissue formation in comparison to plateletrich plasma. Platelets, 29(1), 48-55.

10. Sohn, D. S., Huang, B., Kim, J., Park, W. E., \& Park, C. C. (2015). Utilization of autologous concentrated growth factors (CGF) enriched bone graft matrix (Sticky bone) and CGF-enriched fibrin membrane in Implant Dentistry. J Implant Adv Clin Dent, 7, 11-29.

11. Hägi, T. T., Laugisch, O., Ivanovic, A., \& Sculean, A. (2014). Regenerative periodontal therapy. Quintessence Int, 45(3), 185-192.
12. Cortellini, P., \& Tonetti, M. S. (2015). Clinical concepts for regenerative therapy in intrabony defects. Periodontology 2000, 68(1), 282-307.

13. Sculean, A., Nikolidakis, D., \& Schwarz, F. (2008). Regeneration of periodontal tissues: combinations of barrier membranes and grafting materials-biological foundation and preclinical evidence: a systematic review. Journal of clinical periodontology, 35, 106-116.

14. Fujioka- Kobayashi, M., Miron, R. J., Hernandez, M., Kandalam, U., Zhang, Y., \& Choukroun, J. (2017). Optimized platelet- rich fibrin with the low- speed concept: growth factor release, biocompatibility, and cellular response. Journal of periodontology, 88(1), 112-121.

15. Ghanaati, S., Booms, P., Orlowska, A., Kubesch, A., Lorenz, J., Rutkowski, J., ... \& Choukroun, J. (2014). Advanced platelet-rich fibrin: a new concept for cell-based tissue engineering by means of inflammatory cells. Journal of Oral Implantology, 40(6), 679-689.

16. Bielecki, T., M Dohan Ehrenfest, D., A Everts, P., \& Wiczkowski, A. (2012). The role of leukocytes from L-PRP/L-PRF in wound healing and immune defense: new perspectives. Current pharmaceutical biotechnology, 13(7), 1153-1162.

17. Soni, R., Priya, A., Yadav, H., Mishra, N., \& Kumar, L. (2019). Bone augmentation with sticky bone and platelet-rich fibrin by ridge-split technique and nasal floor engagement for immediate loading of dental implant after extracting impacted canine. National Journal of Maxillofacial Surgery, 10(1), 98-101.

18. Miron, R. J., Fujioka-Kobayashi, M., Hernandez, M., Kandalam, U., Zhang, Y., Ghanaati, S., \& Choukroun, J. (2017). Injectable platelet rich fibrin (i-PRF): opportunities in regenerative dentistry?. Clinical oral investigations, 21(8), 2619-2627. 